\title{
INTRAVESICAL WIRE AS FOREIGN BODY IN URINARY BLADDER
}

\author{
DILIP K. PAL, ASIM K. BAG \\ Departments of Urology and Radiology, Medical College and Hospital, Kolkata, India
}

\begin{abstract}
Foreign bodies in the urinary bladder are frequently the objects of jokes among doctors, but they may sometimes cause serious implications to the patients. Here we present our experiences in 3 such cases where long segments of wire were introduced into the urinary bladder through the urethra.
\end{abstract}

Key words: urinary bladder; foreign bodies; calculus

Int Braz J Urol. 2005; 31: 472-4

\section{INTRODUCTION}

A multitude of foreign bodies have been found in the urinary bladder, such as a needle, a bullet, a safety pin, an animal feather, pieces of candle, a thermometer, chewing gum, a Steinman pin, a gauze pack, a toothbrush, a metal hook, and a scalpel blade etc., as reported in the literature (1-3). In most cases, they are either self introduced to produce erotic sensations and sexual gratification or introduced by someone else to get relief from urinary complaints (2). Here we report 3 cases and their successful management.

\section{CASE REPORTS}

Case 1 - An 18-year-old boy presented with complaints of dysuria, terminal hematuria and suprapubic pain that had lasted for 2 weeks. A hysical examination was unremarkable. Urinalysis showed plenty of pus cells with significant growth of E. coli in the urine culture. An X-ray of the pelvis showed a coiled up radiopaque shadow in the bladder region (Figure-1). Upon further interrogation, the patient admitted that he was used to masturbating by introducing an electric wire through the urethra. One month previous, he had lost the wire inside his erected penis during this process. Under general anesthesia, a 16- inch long electric wire was removed through cystoscope with an uneventful recovery.

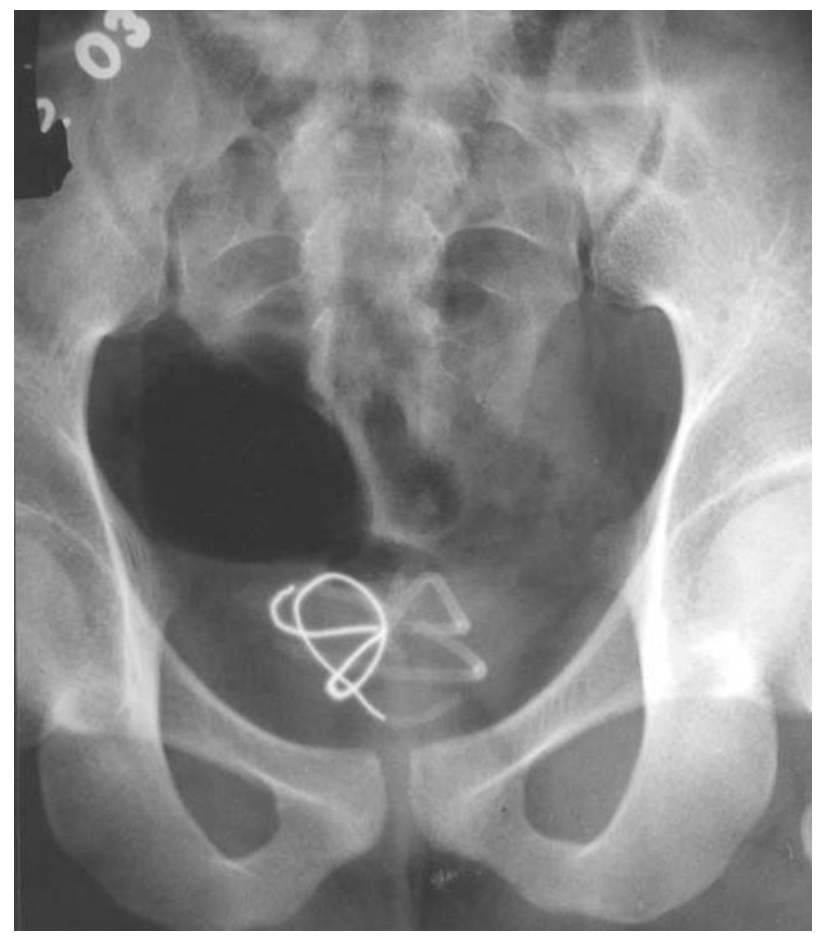

Figure 1-X-ray of the pelvis showing coiled up wire in urinary bladder. 
Case 2 - A 22-year-old boy presented severe burning micturation with poor urinary stream over the past 2 months. He had a history of repeated attacks of urinary tract infections in the last few years. A physical examination revealed meatal stenosis with tenderness in the suprapubic region. Urinalysis showed plenty of pus cells along with red blood cells. Urine culture showed the growth of E. coli $10^{7} \mathrm{CFU} / \mathrm{mL}$. An X-ray of the pelvis suggested a large bladder stone with a coiled up wire inside it (Figure-2). The parents of the patient admitted that in his childhood a quack had introduced an electric wire through his urethra for treatment of meatal stenosis. They did not know whether it had ever been removed or not. The stone was removed by cystolitholapaxy and the wire was removed by cystoscopy after a meatotomy.

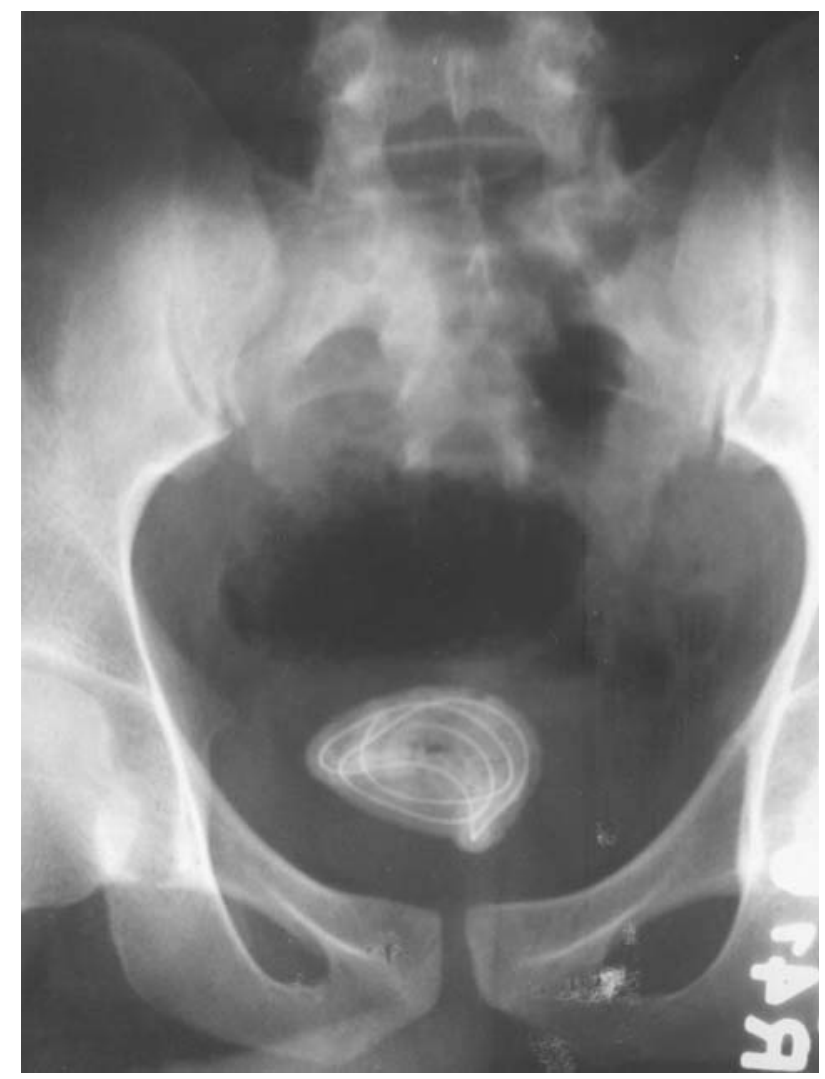

Figure $2-X$-ray of the pelvis demonstrates a coiled up wire within a stone in the urinary bladder.

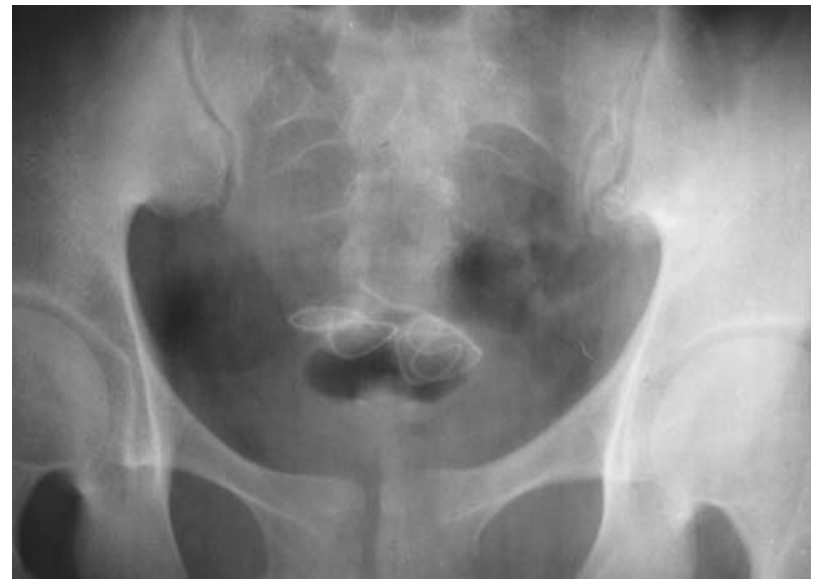

Figure 3 - X-ray of the pelvis showing coiled up wire in the urinary bladder.

Case 3 - A 16-year old boy presented with features of acute cystitis for a previous few days. Clinical examination was unremarkable except for suprapubic tenderness. A urine examination showed plenty of pus and red blood cells. Urine culture showed a growth of Pseudomonas $10^{6} \mathrm{CFU} / \mathrm{mL}$. A pelvic ultrasound showed an echogenic foreign body in the urinary bladder. An X-ray of the pelvis showed a coiled wire in the urinary bladder (Figure-3). On further interrogation, the patient admitted that a wire was introduced by his sexual partner for more sexual gratification and he had lost the tail end of the wire. Cystoscopy confirmed the wire in the bladder, which was removed under general anesthesia.

\section{COMMENTS}

Foreign bodies may find their way into the urinary bladder by accident, deliberate introduction through the urethra or migration from the neighboring organs (1-3). When a wire is introduced through the urethra, part of it remains in the urethra and part goes to the bladder cavity. At the time of micturation, the contracting bladder curled the part of the wire that had partly moved into the bladder from the urethra. Gradually the whole of the wire originally present in the urethra was pulled into the bladder (2). To avoid embarrassment, patients tend to seek treatment late, often waiting until the problem becomes 
symptomatic (3). Usually the patients present with urethritis, cystitis, recurrent UTI, or hematuria (1-3). $\mathrm{X}$-rays are sufficient to diagnose such conditions. Intravenous urography rarely gives any additional information, and is indicated only to diagnose radiolucent objects. Cystoscopy gives the final diagnosis in doubtful cases. Managing the situation seeks to remove the foreign body with minimum trauma, and cystoscopic removal is the ideal approach. Where a stone has formed, it should be broken by litholapaxy or intracorporeal lithotripsy together with the removal of the foreign body. Large foreign bodies may be re- moved by suprapubic cystostomy where endoscopic removal is not possible.

\section{REFERENCES}

1. Pal DK: Intravesical foreign body. Ind J Surg. 1999; 61: 381-3.

2. Eckford SD, Persad RA, Brewster SF, Gingell JC: Intravesical foreign bodies: five-year review. Br J Urol. 1992; 69: 41-45.

3. Kenney RD: Adolescent males who insert genitourinary foreign bodies: is psychiatric referral required? Urology. 1988; 32: 127-9.

Received: April 5, 2005 Accepted after revision: June 20, 2005

Correspondence address:

Dr. Dilip Kumar Pal

Vinayak Garden, Flat No. - A / 3D

41, Simla Road, Kolkata - 700 006, India

E-mail:drdkpal@hotmail.com 